\title{
A implantação de um núcleo de inovação tecnológica: a experiência da Fiocruz
}

\author{
The establishment of a technology transfer office: the experience at Fiocruz
}

\section{La implantación de una unidad de innovación tecnológíca: la experiencia en Fiocruz}

Patricia Seixas da Costa Braga | patriciaseixasdacosta@gmail.com

Fundação Oswaldo Cruz, Presidência. Rio de Janeiro, Brasil.

Lais Silveira Costa | lais.costa@fiocruz.br

Fundação Oswaldo Cruz, Presidência. Rio de Janeiro, Brasil.

\section{Resumo}

A Lei de Inovação foi uma iniciativa governamental para a criação de um ambiente propício à inovação no Brasil. Suas regras impactaram definitivamente o mapa de inserção das instituições acadêmicas e científicas nacionais (instituições científicas e tecnológicas - ICT) nesse processo. Entre suas estratégias, induziu a adequação organizacional das ICT, tornando obrigatória a implantação de núcleos de inovação tecnológica (NIT). Adicionalmente, o novo Marco Legal da Ciência, Tecnologia e Inovação, recentemente sancionado, pode representar um divisor de águas para a atuação das ICT brasileiras, aumentando seu protagonismo. Considerando a importância do papel desempenhado pelos NIT nessa área e a complexidade inerente à sua estruturação e atuação, o estudo que fundamenta o presente artigo se debruçou sobre o caso do NIT da Fundação Oswaldo Cruz (Fiocruz). E, ao identificar estratégias de implantação e resultados observados, as autoras mapearam os principais desafios e orientações, procurando oportunizar uma reflexão capaz de contribuir para a implementação e consolidação de NIT nas ICT brasileiras.

Palavras-chave: instituições de ciência e tecnologia; núcleo de inovação tecnológica; Lei de Inovação; patentes; parcerias tecnológicas. 


\begin{abstract}
The Lei de Inovação (Innovation Law) represented a governmental initiative to create an enabling environment for innovation in Brazil. Its rules affected definitively the insertion map of national academic and scientific institutions (ICT of Portuguese instituições científicas e tecnológicas) in this process. Among its strategies, it sought to induce the organizational adjustment of the ICT, requiring the establishment of technology transfer offices (NIT, of Portuguese núcleos de inovação tecnológica). Furthermore, the new Legal Framework for Science, Technology and Innovation, recently sanctioned, may represent a game changer in the Brazilian ICT performance, increasing their protagonism. Given the importance of the NIT in this area, as well the inherent complexity of its structuring process and the operation of these offices, this article presents a study that looks into the case of the NIT at the Oswaldo Cruz Foundation, known as Fiocruz. Based on the identification of the strategies for implementing that NIT and their results, we mapped out the main challenges and guidelines, so that providing a reflection able to contribute to the implementation and consolidation of the NIT at Brazilian ICT.
\end{abstract}

Keywords: science and technology institutions; technology transfer office; Lei de Inovação (Innovation Law); patents; technological partnership.

\title{
Resumen
}

A Lei de Inovação (Ley de Innovación) representa una iniciativa del gobierno de crear un entorno propicio para la innovación en Brasil. Sus reglas afectaron definitivamente el mapa de inserción de las instituciones académicas y científicas nacionales (ICT del portugués instituciones científicas y tecnológicas) en este proceso. Entre sus estrategias, ella ha inducido la adecuación organizativa de las ICT, tornando obligatoria la implantación de unidades de innovación tecnológica (NIT, del portugués núcleos de inovação tecnológica). Además, el nuevo Marco Legal de la Ciencia, Tecnología y Innovación, recientemente promulgado, puede representar un punto de inflexión para el desempeño de las ICT de Brasil, aumentando su protagonismo. Teniendo en cuenta el importante papel que desempeñan las NIT en esta área y la complejidad de su estructura y funcionamiento, este artículo presenta un estudio que se ha centrado en el caso de la NIT de la Fundación Oswaldo Cruz (Fiocruz). Además, identificando las estrategias de implantación y los resultados observados, el artículo expone los desafíos y orientaciones esenciales, con el objetivo de propiciar una reflexión capaz de contribuir a la aplicación y consolidación de NIT en las ICT brasileñas.

Palabras clave: instituciones de ciencia y tecnología; unidad de innovación tecnológica; Lei de Inovação (Ley de Innovación); patentes; colaboraciones tecnológicas.

INFORMAÇÕES DO ARTIGO

Contribuição dos autores:

Braga, PSC. Responsável pela concepção, delineamento, análise e interpretação de dados, redação, revisão crítica e aprovação da versão final do artigo a ser publicado.

Costa, LS. Contribuiu com o delineamento, análise e interpretação de dados, redação, e revisão crítica do artigo a ser publicado.

Declaração de conflito de interesses: Não há conflito de interesses.

Fontes de financiamento: As autoras agradecem o apoio da Escola Nacional de Saúde Pública Sergio Arouca, da Fiocruz (Projeto ENSP-007-LIV-07-5), e do Conselho Nacional de Desenvolvimento Científico e Tecnológico (Processo CNPq: 405077/2013-0).

Agradecimento/Contribuições adicionais: As autoras agradecem o apoio da Escola Nacional de Saúde Pública Sergio Arouca, da Fiocruz (Projeto ENSP-007-LIV-07-5), e do Conselho Nacional de Desenvolvimento Científico e Tecnológico (Processo CNPq: 405077/2013-0).

Histórico do artigo: Submetido: 23.fev.2016 | Aceito: 27.jul.2016 | Publicado: 23.dez.2016.

Licença CC BY-NC atribuição não comercial. Com essa licença é permitido acessar, baixar (download), copiar, imprimir, compartilhar, reutilizar e distribuir os artigos, desde que para uso não comercial e com a citação da fonte, conferindo os devidos créditos de autoria e menção à Reciis. Nesses casos, nenhuma permissão é necessária por parte dos autores ou dos editores. 


\section{Considerações iniciais}

No mundo globalizado, a eficiência tecnológica de um país - definida pela capacidade de gerar e introduzir inovações no mercado - passou a exercer papel-chave na dinâmica do desenvolvimento econômico nacional. O seu alcance, entretanto, exige a conjugação de vários fatores: maciços investimentos em P\&D, uma base científica consolidada e sua interação com a indústria, estabelecimento de redes e alianças estratégicas, a atuação do Estado como agente promotor e articulador, entre outros. Esta percepção embasou a política industrial de países como Estados Unidos, Japão e França, e mesmo de economias emergentes, como o caso da Coreia do $\mathrm{Sul}^{1}$, cujo sucesso caracteriza o protagonismo do Estado na indução do processo de inovação e desenvolvimento econômico nacional.

No Brasil, esse reconhecimento foi coroado pelo artigo 218 da Constituição Federal de 1988². Por esse dispositivo, entre outras disposições, foi estabelecido que a pesquisa científica básica seria destinatária de tratamento prioritário pelo Estado e a pesquisa tecnológica direcionada, preponderantemente, a desenvolver o sistema produtivo nacional e regional. Visando à sua complementação, diante da percepção da necessidade da efetiva construção de um ambiente favorável à inovação no país, foi sancionada a Lei de Inovação, Lei 10.973/2004³, que buscou criar mecanismos de incentivo à inovação mediante o estímulo à pesquisa cientifica e tecnológica no ambiente produtivo e à promoção da interação entre as instituições de ciência e tecnologia (ICT) nacionais e a indústria.

Esse diploma legal também se preocupou em induzir a adequação das estruturas dessas instituições - no que se refere à gestão da política de inovação, notadamente para o estímulo à proteção das criações, licenciamento, inovação e outras formas de transferência de tecnologia -de forma a propiciar o efetivo aproveitamento dos incentivos então previstos. Para tanto, tornou-se obrigatória a existência de um Núcleo de Inovação Tecnológica (NIT) em toda ICT. A importância da existência e atuação dessas estruturas foi recentemente confirmada pelo Novo Marco Legal da Ciência e Tecnologia e Inovação no país, Lei 13.243/2016 $6^{4}$ que ampliou seu escopo de atuação e buscou atribuir-lhes perenidade - expandiu seu rol de competências mínimas e admitiu a possibilidade de atribuição de personalidade jurídica própria aos NIT -, o que pode representar um divisor de águas para a estruturação dessas atividades nas ICT brasileiras.

A implantação de um NIT, a despeito de um arcabouço jurídico que o torna obrigatório, demanda a adoção de ações e estratégias capazes de propiciar a sua estruturação e atuação, implicando, ainda, no enfrentamento de um conjunto de desafios. Neste momento em que uma nova janela de oportunidades se revela para as ICT, o presente artigo apresenta um estudo de caso sobre a experiência de uma instituição de ciência e tecnologia pública federal na área da saúde, a Fundação Oswaldo Cruz (Fiocruz). Busca-se, desse modo, contribuir com o processo de construção de ambientes favoráveis e de indução à inovação nas ICT brasileiras.

O recorte de análise se justifica, principalmente, por dois fatores. Inicialmente, pelo número expressivo de ICT na área da saúde, quase um terço das existentes no país 5 . Adicionalmente, pela inequívoca relação estabelecida entre a saúde e o desenvolvimento nacional, visto que, a despeito do seu caráter social, a saúde é também um bem econômico e um espaço de acumulação e circulação de capital, intensivo em tecnologias portadoras de futuro, que se destaca por sua participação na formação de emprego e renda nacionais ${ }^{6-8}$

Especificamente quanto à Fiocruz, a sua eleição para objeto do presente estudo ainda se deve ao fato de que, além da sua proeminência no cenário nacional de CT\&I, na época da promulgação da Lei de Inovação essa instituição pertencia a um pequeno grupo de ICT brasileiras que já possuía o seu NIT implantado. Não obstante, o novo marco legal também provocou a reestruturação do seu modelo de gestão da inovação.

Este é um estudo exploratório elaborado a partir de uma revisão bibliográfica narrativa. Entre os documentos científicos que embasam o presente artigo, foram incluídas três dissertações de mestrado da Escola Nacional de Saúde Pública Sérgio Arouca que tiveram como objeto a estruturação e atuação da área de gestão de tecnologia na Fiocruz, realizadas no período 2004 a 20119-11. Adicionalmente, foi realizado o levantamento das 
principais normas internas que amparam a estruturação e funcionamento do NIT da Fiocruz ${ }^{12-17}$, e de relatórios institucionais publicados entre os anos de 1999 a 2015 - que correspondem, em sua maioria, a relatórios de gestão e de atividades da Fiocruz e informações extraídas de seu portfólio de inovação ${ }^{18-29}$.

A leitura desses trabalhos e documentos permitiu identificar os principais marcos de implantação dessas atividades na Fiocruz, correlacioná-los com o cenário nacional e internacional e assinalar a interconexão entre atores críticos. Além disso, possibilitou o mapeamento das estratégias adotadas (entre as quais, opções normativas, formação e capacitação de equipe, captação de recursos e a implantação de procedimentos), orientações e principais desafios. Para identificar os possíveis impactos decorrentes da estruturação dessas atividades foram aferidos parâmetros, tais como publicações científicas institucionais em revistas indexadas, depósito de pedidos de patente e o seu licenciamento a terceiros para, por fim, avaliar e refletir sobre os resultados até então alcançados. Com isso espera-se contribuir para a implementação e consolidação de NIT nas ICT brasileiras.

\section{A gestão de tecnologia na Fiocruz: marcos da implantação}

A estrutura, a organização e o formato de gestão de tecnologia na Fiocruz passaram por um longo processo de construção. As primeiras estratégias relacionadas à proteção patentária e à transferência de tecnologia datam de meados da década de 1980, época de acirradas discussões voltadas para a reformulação do sistema internacional de propriedade intelectual, cujos resultados foram de grande impacto para o setor de saúde de países em desenvolvimento como o Brasil.

Naquela década, a legislação brasileira vigente, utilizando-se do elevado grau de liberdade para a regulamentação da matéria, ainda incluía no rol de invenções não patenteáveis "as substâncias, matérias, misturas ou produtos químico-farmacêuticos e medicamentos, de qualquer espécie, bem como os respectivos processos de obtenção ou modificação" ${ }^{\circ}$. O mercado brasileiro era, então, livre de monopólios reconhecidos pelo Estado com relação a um grande número de inovações da área da saúde, e o não reconhecimento do privilégio patentário a esses setores tecnológicos fazia parte da política brasileira de incentivo à indústria nacional.

Entretanto, a expansão das relações comerciais internacionais, promovida pelos avanços tecnológicos obtidos a partir da década de 1970, fez com que fosse pleiteada, em 1986, a inclusão das discussões de revisão do sistema internacional de propriedade intelectual na pauta de discussão da Rodada Uruguai do Acordo Geral sobre Tarifas e Comércio - Gatt (General Agreement on Tariffs and Trade). Tais reivindicações tinham em vista atender, em especial, aos interesses da indústria norte-americana, incluindo os de fabricantes de produtos químicos, farmacêuticos e biotecnológicos ${ }^{31}$. Tinha como uma de suas principais finalidades promover o estabelecimento de padrões mínimos de concessão de patentes a serem observados por todos os Estados, o que incluía a fixação de prazos de concessão, a restrição à discriminação de setores tecnológicos, entre outros. Apesar da resistência de vários países, a inclusão pleiteada foi realizada em 1989, após a concordância do Brasil e da Índia ${ }^{32}$.

Qualquer alteração no cenário internacional relacionada à normatização da matéria poderia resultar em reflexos diretos no acesso a tecnologias da área da saúde e, consequentemente, influenciar as atividades desenvolvidas pela Fiocruz. Essa conjuntura embasou o processo de estruturação das atividades de gestão de tecnologia nessa instituição, que, em 1986, criou o Núcleo de Estudos Especiais da Presidência (NEP), cujo objetivo incluía o incentivo à articulação e integração das atividades internas de ciência e tecnologia com o conjunto de políticas de saúde, o apoio aos processos de concessão de patentes e à transferência de tecnologia das inovações geradas internamente, de forma a viabilizar a sua transformação em benefícios concretos para a população.

Em 1990, ano seguinte ao da efetiva inclusão da discussão sobre a regulamentação internacional referente à propriedade intelectual na rodada de negociações do Gatt, o NEP sofreu um processo de 
reformulação e deixou de ser apenas um núcleo de estudos, passando a constituir a Coordenação de Gestão Tecnológica (Gestec), o que inaugurou a implantação de atividades direcionadas à proteção dos resultados das pesquisas desenvolvidas por institutos de propriedade industrial e a implementação de ações voltadas para a transferência de tecnologia na Fiocruz.

No cenário internacional, como resultado da criação da Organização Mundial do Comércio (OMC) e da assinatura do Acordo sobre Aspectos dos Direitos de Propriedade Intelectual Relacionados ao Comércio (TRIPs), em 1994, foi assegurada a ampliação do escopo de proteção nacional mínima a ser conferida pela concessão de patentes, com especial destaque para a proibição aos Estados da discriminação de certos segmentos do setor tecnológico ${ }^{7}$. Os países membros da então criada OMC deveriam ajustar as suas legislações para torná-las compatíveis com as novas regras internacionais. Com essa finalidade, o Brasil promulgou a Lei de Propriedade Industrial, Lei 9.279/199633, e revogou o Código de Propriedade Industrial em vigor.

No mesmo ano, buscando acompanhar as transformações ocorridas no sistema internacional de propriedade intelectual e na legislação nacional, notadamente no que diz respeito à concessão de patentes, a Fiocruz instituiu a sua política de propriedade intelectual, até hoje vigente: estabeleceu diretrizes e disciplinou sobre matérias como titularidade de invenções, processo decisório e responsabilidade pelo depósito e tramitação de pedidos de patente, exploração de tecnologias, repartição de benefícios com inventores e sigilo ${ }^{14}$. Além disso, criou mecanismos voltados para proteger o patrimônio científico e tecnológico, estimular processo inovador, viabilizar o retorno do investimento em $\mathrm{P} \& \mathrm{D}$, fortalecer e ampliar a capacitação tecnológica na casa. Também fixou e delimitou a área de atuação da Gestec, que então já correspondia ao NIT posteriormente preconizado pela Lei de Inovação e pelo novo Marco Legal da Ciência e Tecnologia e Inovação no Brasil. Em seguida, em 1998, ainda como efeitos da promulgação da Lei 9.279/199633, a Fiocruz transformou a Gestec numa assessoria da sua Presidência. A consolidação e perenidade dessa estrutura foram posteriormente sacramentadas pelo Regimento Interno da Fiocruz ${ }^{12}$.

Assim, a gestão da propriedade industrial e da transferência de tecnologia na Fiocruz era matéria de competência da Presidência. Entretanto, antes mesmo da promulgação da Lei de Inovação, duas das unidades da Fiocruz - que, além de executar atividades de P\&D, fabricam produtos voltados para o atendimento de demandas do Ministério da Saúde - deram início a um movimento voltado para a estruturação de suas áreas especializadas sobre a matéria. Essas unidades, Biomanguinhos e Farmanguinhos, a partir de 1998, passaram a adquirir tecnologia como estratégia para ampliar o leque de produtos fornecidos para o atendimento da demanda dos programas de saúde pública do governo federal. Tal fato implicou na necessidade da ampliação da capacidade interna de negociação dos contratos para a aquisição das tecnologias e deflagrou o processo de organização dessas unidades.

Além disso, a partir de 2002, várias iniciativas foram tomadas pela Fiocruz buscando induzir a introdução no mercado de inovações oriundas das pesquisas realizadas, com destaque para a criação do Programa de Desenvolvimento Tecnológico em Insumos para a Saúde/PDTIS; Programa de Desenvolvimento Tecnológico em Saúde Pública/PDTSP; o desenvolvimento e implantação do Projeto do Centro de Desenvolvimento Tecnológico em Saúde/CDTS ${ }^{10}$. Essas iniciativas implicaram alteração da percepção sobre a importância estratégica da gestão da propriedade industrial e da transferência de tecnologia para o alcance dos anseios institucionais, que foi intensificada com a entrada em vigor da Lei de Inovação, em 2004. O consequente aumento da demanda interna implicou na dificuldade do seu atendimento no formato centralizado de gestão até então em vigor. Assim, ações voltadas para a implantação de competências locais foram deflagradas.

Nesse contexto, em 2006, a partir da compreensão de que apenas mediante uma maior aproximação da gestão central com a pesquisa realizada dentro de cada unidade seria possível atender satisfatoriamente às demandas internas e promover a gestão integrada das tecnologias geradas, sem perder a unidade e coesão institucional em torno do tema, a Fiocruz concebeu o Sistema Gestec-NIT. A sua posterior implantação, a partir de 2007, inaugurou novo formato de gestão da propriedade intelectual e 
transferência de tecnologia na instituição. Representou um esforço de coordenação e organização das diversas iniciativas das unidades da Fiocruz, reconhecendo-lhes competência para gerir internamente uma parte predefinida das questões que fossem associadas ao tema, mas mantendo algumas atividades como de competência exclusiva da área central. Além disso, tinha como finalidade adequar a política institucional de propriedade intelectual e transferência de tecnologia à da Lei de Inovação, bem como expandir e aprimorar o atendimento às demandas institucionais zelando pela integração das ações e atividades executadas na Fiocruz.

Com essa finalidade, a implantação do Sistema Gestec-NIT demandou o estabelecimento de uma estrutura especializada em propriedade intelectual e transferência de tecnologia em cada uma de suas unidades, os chamados Núcleos de Inovação Tecnológica de Unidade Técnico-Científica (NIT-UTC). A Gestec seria o núcleo central desse sistema, responsável por coordená-lo e por promover a interligação e articulação entre os NIT-UTC criados, que no total hoje correspondem a 20 núcleos ${ }^{20}$. Sua implantação não acarretou a duplicação ou sobreposição de estruturas - a Gestec continuou atuando nas suas áreas de competência e a cada NIT foram delegadas atribuições específicas, compatíveis com o perfil de sua Unidade Técnico-Científica. As demandas originalmente encaminhadas pelos pesquisadores das diversas unidades da Fiocruz diretamente à Gestec passariam a ser primeiramente submetidas aos NIT-UTC, que atenderiam aquelas possíveis de serem respondidas e encaminhariam ao núcleo central do sistema implantado aquelas que fugissem a sua esfera de ação ${ }^{10,11}$.

A criação e implantação desse novo modelo de gestão precisaram respeitar a configuração estrutural e especificidades da Fiocruz, tais como, seu processo de formação, a heterogeneidade de suas unidades, autonomia, variedade de competências, sua conformação político-decisória ${ }^{20}$. Por essa razão não seguiu um padrão e cada um dos NIT foi sendo moldado em razão das características de sua unidade.

Em resumo, nos últimos 30 anos, o processo de construção e consolidação das atividades de gestão de tecnologia na Fiocruz foi diretamente influenciado pelo contexto de transformações no cenário nacional e internacional, podendo ser sistematizado em cinco marcos de evolução, conforme se observa na Figura 1.

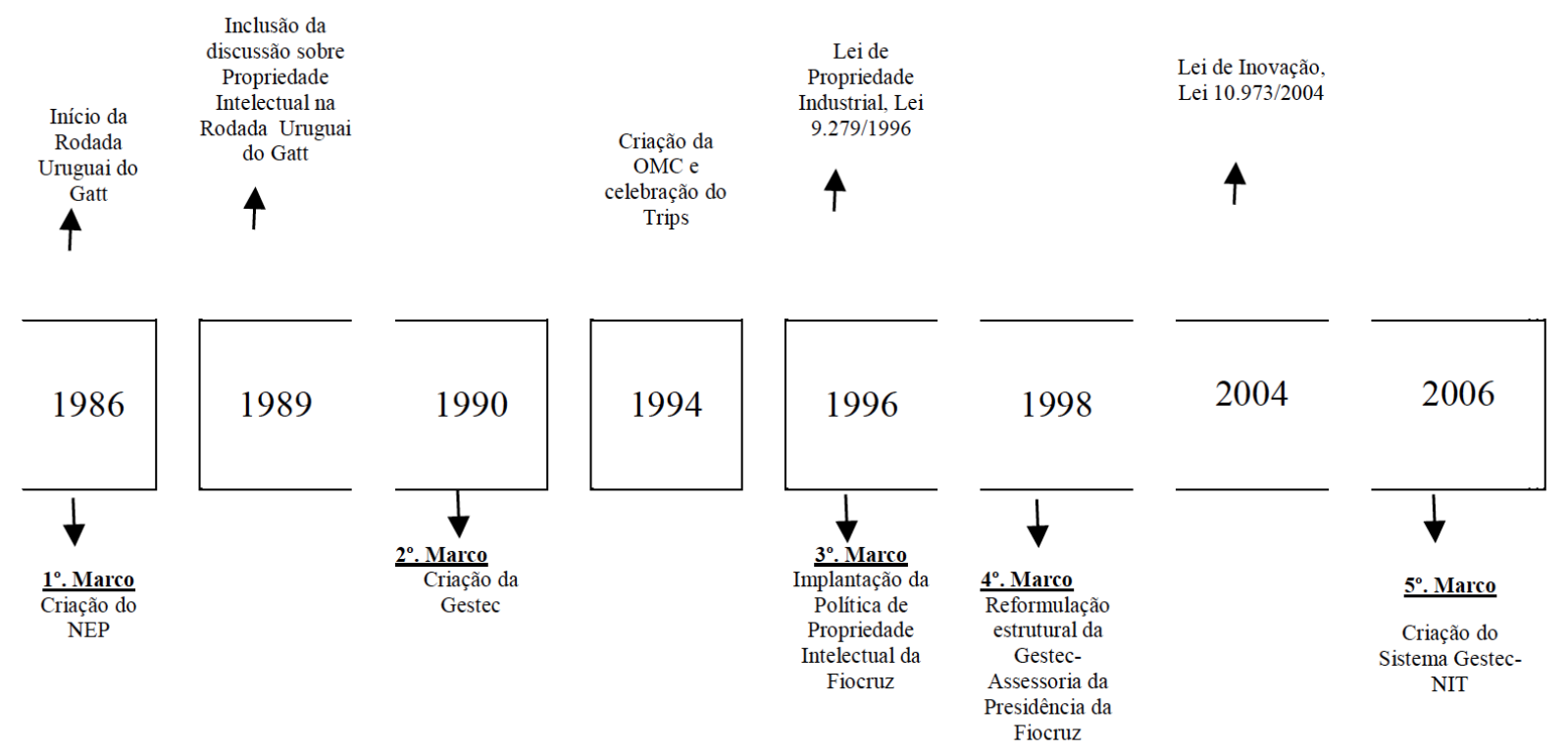

Figura 1 - Marcos de implantação da gestão de tecnologia na Fiocruz Fonte: Elaborada pelas autoras (2016). 


\section{Estruturação das atividades de gestão da propriedade intelectual e transferência de tecnologia: estratégias adotadas pela Fiocruz}

O processo de estruturação das atividades de gestão da propriedade intelectual e transferência de tecnologia na Fiocruz demandou o estabelecimento de um conjunto de estratégias e o enfrentamento de desafios de várias ordens.

No que se refere à regulamentação interna, a edição de portarias embasou os grandes marcos de implantação e consolidou a política voltada para a gestão da inovação. Seu processo de elaboração, antes da criação do Sistema Gestec-NIT, era relativamente simples - as propostas eram discutidas na Presidência da Fiocruz, traduzidas em portarias e então sancionadas. Após sua criação, por intermédio dos NIT, passou a demandar consulta às suas unidades, processo que, apesar de garantir uma maior aderência às normas editadas, é mais complexo e longo. Como resultado, a revisão e atualização da política de propriedade intelectual e transferência de tecnologia da Fiocruz à luz da Lei de Inovação não foi ainda finalizada. Oportunidades como o compartilhamento de laboratório e critérios para o pagamento de bolsas de incentivo à inovação, decorrentes de parcerias estabelecidas com empresas, e prestação de serviços, entre outras, não chegaram a ser normatizadas na instituição.

Quanto ao suporte técnico e administrativo ao depósito, acompanhamento e tramitação de pedidos de patente no Brasil e no exterior e, ainda, para o subsidio à negociação e elaboração de contratos de transferência de tecnologia, uma importante estratégia adotada foi a contratação de serviços especializados em propriedade intelectual. Note-se que, para a Fiocruz, esses serviços sempre constituíram uma ferramenta de gestão imprescindível, uma vez que a contratação de escritórios no exterior enfrenta problemas como insegurança para a seleção técnica do contratado e incompatibilidade entre os procedimentos obrigatórios à celeridade demandada para a tramitação de pedidos de patente fora do país. Assim, o escritório contratado no Brasil, além de possuir uma rede de relacionamento com escritórios ao redor do mundo capazes de atenderem à Fiocruz em qualquer país que seja necessário, passou gradativamente a ser o responsável pela própria contratação e pelo pagamento dos serviços prestados no exterior.

A formação de equipe também demandou o estabelecimento de estratégias. Inicialmente, a Fiocruz contou com especialistas oriundos do INPI e da Finep, bolsistas e terceirizados ${ }^{9}$. A sua consolidação, entretanto, só foi possível em razão dos concursos realizados a partir de 1996, quando passaram a ser previstas vagas de analistas com perfil específico para atuação na área de propriedade intelectual. No final de 1998, o NIT da Fiocruz possuía uma equipe técnica que contava com oito pessoas, número este que subiu para 12 em 2004, ano da promulgação da Lei de Inovação, chegando a aproximadamente 76 profissionais no final de 2015: 22 atuando na Gestec e 54 no Sistema Gestec-NIT ${ }^{18-20,34}$.

A despeito dos concursos específicos realizados, o remanejamento de servidores de outras áreas, terceirizações, e contratações de bolsistas ainda correspondem a uma parcela significativa da sua força de trabalho, uma estratégia até hoje utilizada para a manutenção e ampliação das atividades desenvolvidas. Além disso, apesar do salto quantitativo, boa parte desses profissionais acumula funções dentro da própria Fiocruz, ou seja, não atuam de forma exclusiva nos seus NIT ${ }^{20}$. Tal fato sugere que ainda persiste certa carência na percepção institucional quanto à prioridade das suas atividades, denota a fragilidade desses NIT e, consequentemente, do próprio sistema implantado.

Além disso, a consolidação e expansão da equipe demandou um grande esforço de capacitação, para o que se valeu da utilização de expertises internas e de parceria estabelecida com diversos atores, no Brasil e no exterior, merecendo destaque a realizada com o INPI ${ }^{9}$. Foram, assim, promovidos estágios, cursos e treinamentos - por exemplo, o de monitoramento tecnológico e de busca -, e organizados eventos diversos, com destaque para ciclos de debates e simpósios, entre outras iniciativas ${ }^{35-37}$. Esses esforços de capacitação, além de se destinarem aos profissionais da Gestec e do Sistema Gestec-NIT, tinham por finalidade fomentar 
a cultura institucional, buscando promover uma maior proximidade com a comunidade científica e afastar eventuais resistências às questões inerentes à propriedade intelectual e transferência de tecnologia. Ademais, procurou proprocionar o debate sobre temas estratégicos associados ao processo e à promoção de inovação na saúde, e, ainda, propiciar a aproximação e articulação com representantes do setor produtivo.

Quanto aos aspectos financeiros, o orçamento institucional cobre as atividades de rotina, devendo ser destacado que o custeio do portfólio de patentes é centralizado, ou seja, é responsabilidade da sua Presidência. Para além disso, ações de expansão, consolidação e fortalecimento têm sido deflagradas principalmente a partir da captação de recursos junto às agências de fomento. $O$ próprio processo de implantação do Sistema Gestec-NIT foi impulsionado pela aprovação de projeto submetido à Finep em 2006 - que tinha como proposta estabelecer um NIT-UTC piloto e, a partir dessa experiência, implantar gradativamente os demais NIT-UTC na Fiocruz ${ }^{10}$ - e cuja execução foi de grande importância para a sua consolidação. Recentemente, a Fiocruz teve um projeto contemplado por uma chamada pública aberta pelo CNPq, no momento em execução, e cujo objetivo é fortalecer do trabalho dos NIT nessa instituição, com destaque para a implantação de novas competências, no caso a incorporação da sistemática de elaboração de estudos de viabilidade técnico-econômica (EVTE) para avaliação de tecnologias nela geradas. Como resultado, espera-se dar um salto qualitativo nas atividades desenvolvidas pelo Sistema Gestec-NIT. Há que se destacar, entretanto, que a dependência de captações externas para a expansão, consolidação e fortalecimento denota também certa fragilidade do sistema implantado ${ }^{38}$.

Ademais, quanto às estratégias para a implantação de procedimentos institucionais, a despeito de todas as iniciativas até então tomadas, pouca coisa está regulamentada ou claramente definida. Na sua maioria, são procedimentos que se direcionam ao depósito e tramitação de pedidos de patente que contam com uma instância decisória, a Comissão de Patentes (Copat), criada em 2002. Outro procedimento regulamentado corresponde ao processo de registro de programas de computadores, o que foi levado a efeito em 2013. Além disso, a implantação do Sistema Gestec-NIT demandou a criação de um procedimento específico para a tramitação de demandas entre os NIT e a Gestec, indispensável para o registro e acompanhamento das solicitações e casos em andamento, recentemente ampliado, aperfeiçoado e transformado em um sistema online. No entanto, falta conhecimento, clareza e, principalmente, integração entre os processos de trabalho executados por cada uma das áreas técnicas do próprio NIT central do Sistema Gestec-NIT, o que sugere a necessidade de reformulação dos processos internos, notadamente para a promoção de uma maior conexão entre as atividades executadas. Tal fato dificultaria a descentralização das suas atividades para os seus NIT e comprometeria a sua própria atuação $0^{10}$. Adicionalmente, diante da constatação da falta de conhecimento da comunidade científica a respeito dos procedimentos que precisam ser observados, seja para a promoção da proteção do resultado de suas pesquisas, ou para o estabelecimento de parcerias e outros temas relacionados com a gestão da inovação, foi publicado em 2015 um guia de ações preventivas para orientar a comunidade científica e promover a sua aproximação com os NIT ${ }^{39}$.

Por fim, a implantação do Sistema Gestec-NIT demandou a instituição de um comitê gestor, uma instância colegiada representativa das unidades científicas e unidades técnicas da Fiocruz, que, coordenado pela Gestec, tinha por objetivo original criar e implantar os NIT em cada uma das unidades técnicas e responsabilizar-se pela operação do sistema. Esse comitê, em funcionamento até a presente data, se caracteriza como uma instância de planejamento, gestão e de integração do sistema implantado, para o que realiza quatro reuniões ordinárias por ano e extraordinárias sempre que necessário ${ }^{20}$. 


\section{Dos impactos decorrentes da implantação do Sistema Gestec-NIT}

Uma reflexão crítica, mas não conclusiva, sobre as estratégias adotadas pela Fiocruz pode ser embasada em algumas variáveis objetivas: relação entre o número de publicações em revistas indexadas e o número de depósitos de pedidos de patente; e a evolução anual do número de contratos de licenças de patente celebrados.

Sendo a Fiocruz uma instituição pública, comprometida com a difusão do conhecimento, as tecnologias geradas internamente são, regra geral, traduzidas em artigos científicos. Assim, no que se refere ao primeiro conjunto de variáveis, busca-se em números a confirmação do pressuposto de que a implantação de um NIT em cada unidade da Fiocruz seria capaz de promover a aproximação entre a comunidade científica e as atividades de gestão de tecnologia, o que facilitaria e promoveria o acesso, aos seus pesquisadores, dos mecanismos institucionais disponíveis para a proteção dos resultados das pesquisas realizadas e implicaria no incremento do número de tecnologias protegidas pela Fiocruz.

Ainda quanto a esse primeiro conjunto de fatores, cabe ressaltar que um pedido de patente depositado em um determinado território pode ser desdobrado em solicitações de patente em diversos países ao redor do mundo. Tal fato implica na constatação de que considerar o total de pedidos de patente depositados por uma instituição enseja computar mais de uma vez a proteção para uma mesma tecnologia. Assim, para identificar quantas tecnologias estão protegidas há que se contabilizar apenas o depósito prioritário, ou seja, o primeiro depósito de pedido de patente realizado para uma determinada tecnologia. Nesse contexto, uma vez que os pedidos prioritários da Fiocruz são depositados no Brasil, a identificação de quantas novas tecnologias foram protegidas por ano deve se restringir aos pedidos depositados nesse país, razão pela qual não foi apontado o número de solicitações realizadas no exterior.

Assim, o Gráfico 1 apresenta o número de depósitos de pedidos de patente realizados pela Fiocruz no Brasil, o Gráfico 2 o número de publicações em revistas indexadas realizadas pela Fiocruz, e ambos englobam os anos de 2001 a 2014.

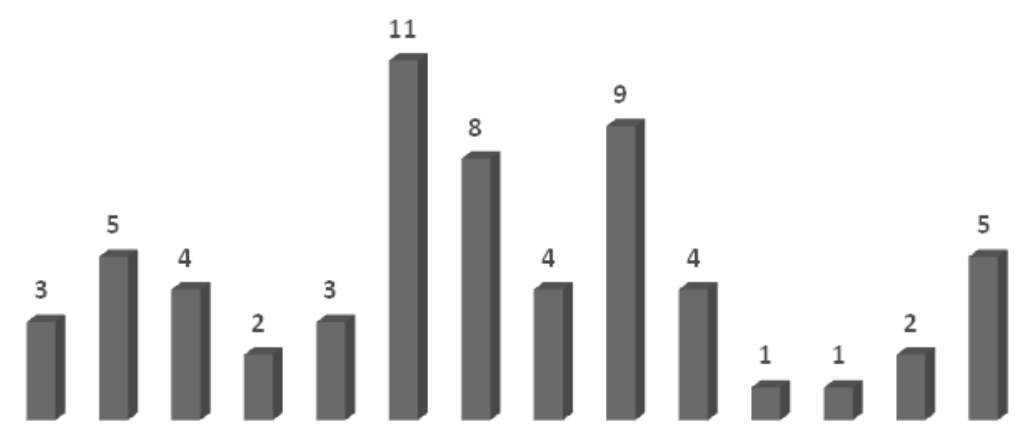

20012002200320042005200620072008200920102011201220132014

Gráfico 1 - Depósito de pedidos de patente no Brasil - Fiocruz

Fonte: Adaptado, pelas autoras, de relatórios de gestão Fiocruz (2008; 2009; 2010; 2011; 2012; 2013; 2014) ${ }^{21-27}$; e do relatório de atividades Fiocruz $(2012)^{28}$. 


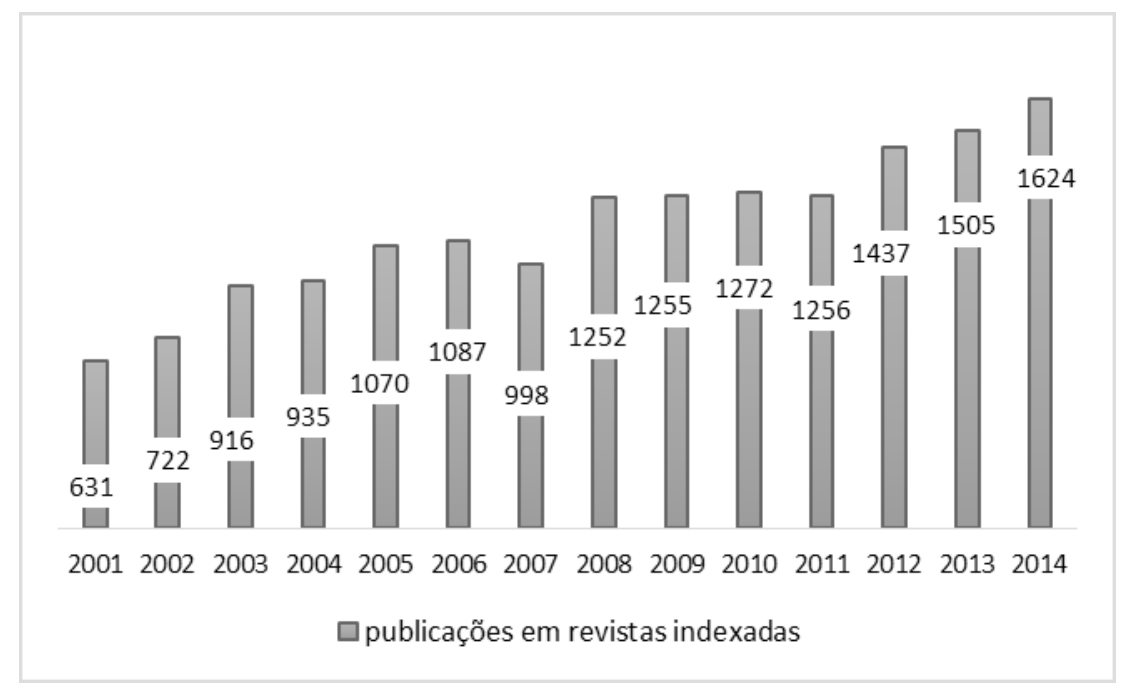

Gráfico 2 - Publicações da Fiocruz em revistas indexadas

Fonte: Adaptado, pelas autoras, de relatórios de gestão Fiocruz (2008; 2009; 2010; 2011; 2012; 2013; 2014) ${ }^{21-27}$.

Ao observar os dados acima, é possível constatar que o número de depósitos de patentes por ano pela Fiocruz é oscilante e não segue um padrão linear. A sua produção científica, de forma contrária, vem crescendo gradativamente, ano a ano, salvo uma pequena variação negativa nos anos de 2007 e 2011. Esses números sugerem, a princípio, não só um descompasso entre o número de patentes e a evolução da produção científica da Fiocruz, mas também a falta de correlação entre esses dados, explicada, em parte, pelas peculiaridades do processo que leva ao depósito de pedidos de patente. Entretanto, para se ter uma avaliação mais conclusiva, outras variáveis precisam ser analisadas. É necessário, por exemplo, identificar o número de notificações de invenções recebidas pelo Sistema Gestec-NIT, visto que o número de depósitos anuais no INPI não significa necessariamente que a demanda tenha ou não ocorrido. Sobre esse aspecto deve ser destacado que a Fiocruz possui um rigoroso processo de avaliação de suas invenções - anterior à própria criação do Sistema Gestec-NIT - que visa à otimização de recursos institucionais. Nada é depositado, seja no Brasil ou no exterior, sem a aprovação prévia da sua Comissão de Patentes (Copat), que, além do atendimento dos critérios de patenteabilidade de toda invenção que lhe é submetida, avalia questões relacionadas ao seu custo/oportunidade.

Além disso, visto o boom de depósitos realizados nos anos de 2006 e 2007, há indícios de que a publicação da Lei de Inovação, em 2004, de fato induziu o incremento da demanda pela proteção patentária na Fiocruz. Entretanto, o patamar alcançado não foi mantido e o número de depósitos continuou oscilante, caindo drasticamente a partir de 2010 e esboçando uma retomada em 2014. Esse tópico merece, assim, uma avaliação mais detalhada capaz de embasar o entendimento das razões dessa inconstância e, além disso, o porquê do incipiente número de depósitos de pedidos de patente realizados pela Fiocruz no Brasil.

Outro aspecto importante é a análise dos impactos produzidos pela gestão das tecnologias patenteadas, para a qual é preciso identificar quais dessas tecnologias foram objeto de parcerias, ou seja, quais estão em desenvolvimento ou já se transformaram em produtos introduzidos no mercado. Com esta preocupação, o Gráfico 3 consolida o quantitativo de contratos celebrados pela Fiocruz que envolvem o licenciamento de patentes, ano a ano, a partir de 1999. 


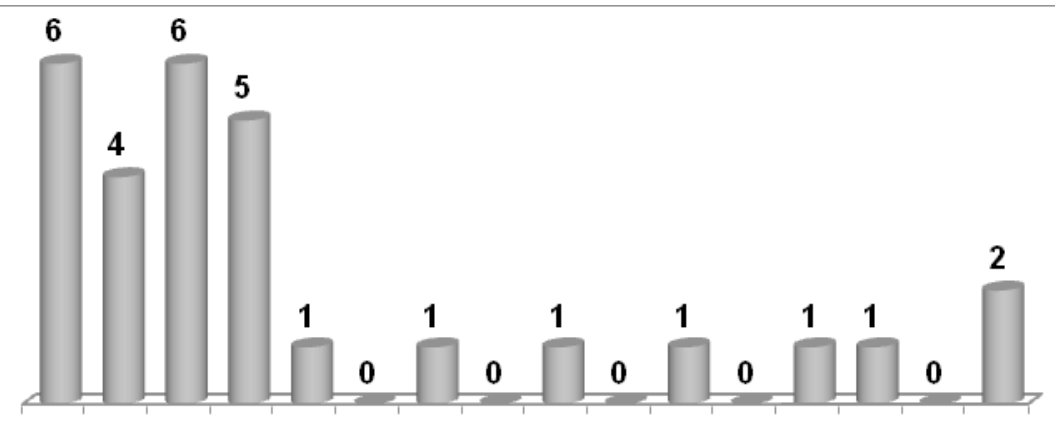

1999200020012002200320042005200620072008200920102011201220132014

Gráfico 3 - Contratos de licença de direitos patentários de titularidade da Fiocruz (1999-2014)

Fonte: Adaptado, pelas autoras, de relatórios de gestão Fiocruz(2008; 2009; 2010; 2011; 2012; 2013; 2014) ${ }^{21-27}$ e do relatório de atividades Fiocruz (2012) ${ }^{28}$; Relatório de atividades Fiocruz/Gestec (1999; 2005) ${ }^{18-19}$.

No período de 1999 a 2005 foram assinados 23 contratos delicença de patente, sendo a maior concentração entre os anos de 1999 e 2002. Após a implantação do Sistema Gestec-NIT, levando-se em consideração o período de 2006 a 2014, foram assinados apenas seis contratos de licenciamento. Apesar da grande diferença numérica, há que se destacar que dos 23 contratos assinados no período de 1999 a 2005, 19 correspondem ao licenciamento, sem exclusividade, de uma única tecnologia, no caso, produção da vela de andiroba, um repelente de mosquitos. Este produto correspondia a uma tecnologia sem alta complexidade e não demandava investimentos para o seu desenvolvimento por parte dos licenciados - a Fiocruz efetivamente transferia a tecnologia de fabricação do produto licenciado. Adicionalmente, seu processo de produção era simples e não demandava complexas estruturas fabris, tendo sido, assim, efetivamente produzido e comercializado pelas empresas licenciadas. Ao contrário, os demais licenciamentos concedidos pela Fiocruz demandam do parceiro licenciado elevados investimentos e tempo para o desenvolvimento do produto. Assim, atualmente existem três produtos em estágio avançado de desenvolvimento: um bioinseticida, que deve ser lançado ainda em 2016; uma vacina contra esquistossomose, em fase II de testes clínicos; e um utensílio para alimentação de bebês de risco. Entretanto, após a vela de andiroba, nenhum outro produto oriundo das patentes licenciadas pela Fiocruz foi introduzido no mercado por um parceiro licenciado.

O aprofundamento desta análise demanda a identificação de outros formatos de parceria celebradas com o setor produtivo, em especial, aquelas que implicam na execução conjunta de projetos em estágios preliminares de desenvolvimento de uma determinada tecnologia e não estabelecidas a partir do licenciamento de patentes concedidas ou de pedidos de patente de titularidade da Fiocruz.

Sobre esse aspecto, a existência de gargalos para o estabelecimento de parcerias com o setor produtivo foi evidenciada em estudo que apontou a necessidade de mudanças organizacionais, culturais e o aprimoramento das ferramentas de gestão direcionadas para a aproximação da instituição com o setor produtivo, com destaque para o licenciamento de patentes de titularidade da Fiocruz e cooperações estabelecidas para o desenvolvimento conjunto de produtos visando introduzir no mercado inovações oriundas das pesquisas por ela realizadas ${ }^{10}$. Para solucionar este problema, uma das iniciativas adotadas foi a organização do Portfólio de Inovação da Fiocruz, uma ferramenta online que visa à difusão de tecnologias geradas, voltadas para a prospecção de parcerias, orginalmente disponibilizada em 2011 e que teve a sua primeira revisão concluída em $2015^{29}$. Os resultados da divulgação desse portfólio, todavia, ainda não introduziram impacto significativo no número de parcerias estabelecidas, visto que após a sua publicação apenas quatro licenciamentos foram assinados.

Além disso, outro estudo ${ }^{11}$ conduzido buscou aprofundar a compreensão sobre as dificuldades inerentes à transformação dos resultados obtidos pelos projetos inseridos no Portfólio de Inovação da Fiocruz em 
produtos. Tal trabalho apontou como barreiras à interação entre a Fiocruz e o setor produtivo a ênfase na incorporação de tecnologia - em detrimento das outras modalidades de transferência de tecnologia -, a ausência da sistematização para oferta de parcerias e da gestão de projetos. Por fim, concluiu que a falta de diretrizes institucionais claras, definidas, com metas e prazos a serem perseguidos e instâncias de fiscalização legitimadas, acarretariam um conjunto de resultados indesejados, entre os quais estariam incluídos a paralização de projetos patenteados, o custeio de patentes nacionais e internacionais sem que as mesmas sejam licenciadas para empresas/setor produtivo, a desarticulação entre o depósito de patentes e a publicação de artigos científicos ${ }^{11}$.

\section{Considerações finais}

No Brasil, a Constituição Federal de $1988^{2}$ e a legislação posteriormente promulgada, com destaque para a Lei de Inovação ${ }^{3}$ e o Novo Marco Legal de Ciência, Tecnologia e Inovação ${ }^{4}$, reconhecem a importância da interação entre a base científica e o setor industrial nacionais para o atendimento das complexas demandas tecnológicas do Sistema Nacional de Inovação e do papel do Estado como agente indutor e articulador. Cabe ressaltar que uma das estratégias adotadas pelo legislador foi induzir a adequação organizacional das ICT brasileiras, o que tornou obrigatória a implantação de estruturas voltadas para a gestão da inovação nessas instituições, os NIT.

Buscando melhor compreender o processo de implantação e gestão dessas estruturas, este artigo apresenta estudo de caso sobre a Fiocruz, que teve o processo de construção do seu NIT deflagrado a partir do final da década de 1980, diretamente influenciado por transformações ocorridas no cenário econômico internacional e nacional - notadamente no que se refere a regras e mecanismos de apropriação de invenções -, cujos reflexos tiveram repercussão de forma particular na área da saúde. Seu processo de implantação demandou a elaboração de normas internas, a formação e capacitação de equipe, a implantação de procedimentos, a disponibilização de recursos financeiros e enfrentou um ambiente cultural nem sempre favorável, entre outros. Além disso, a Lei de Inovação impulsionou a transformação do modelo de gestão do seu NIT já implantado, que passou de um formato centralizado para um modelo sistêmico e descentralizado, o Sistema Gestec-NIT.

A despeito de todas as iniciativas tomadas e os esforços empreendidos para a estruturação, capacitação e o fortalecimento das atividades da gestão da propriedade intelectual e promoção das atividades de transferência de tecnologia pela Fiocruz - não obstante a pujança das pesquisas conduzidas por esta instituição -, os resultados levantados pelo presente estudo sugerem que a lacuna institucional existente entre a bancada e o mercado ainda não foi superada.

A compreensão das razões que têm impedido ou dificultado a obtenção de resultados mais positivos demanda avanços nos estudos sobre o tema, em especial no que se refere à ampliação do entendimento sobre as estratégias adotadas e os desafios enfrentados pelas ICT brasileiras para a estruturação de seus NIT, a análise qualitativa dos resultados obtidos pelas ICT no Brasil - a partir da edição da Lei de Inovação -, e a avaliação das potencialidades proporcionadas pelo novo Marco Legal de Ciência, Tecnologia e Inovação para o aperfeiçoamento e efetividade das atividades de gestão da inovação realizadas pelas ICT nacionais.

\section{Referências}

1. Matias-Pereira J, Kruglianskas I. Gestão de inovação: a Lei de Inovação tecnológica como ferramenta de apoio às políticas industrial e tecnológica do Brasil. Rae Eletr [Internet]. 2005 jul./dez. [citado 2016 nov. 11]; 4(2). Disponível em: http://www.scielo.br/pdf/raeel/v4n2/v4n2a03.pdf

2. Brasil. Constituição da República Federativa do Brasil de 1988. [Internet]. Braślia (DF); 1988 [citado 13 de dezembro de 2016]. Disponível em: https://www.planalto.gov.br/ccivil_03/constituicao/constituicao.htm 
3. Brasil. Lei no 10.973, de 2 de dezembro de 2004. Dispõe sobre incentivos à inovação e à pesquisa científica e tecnológica no ambiente produtivo e dá outras providências. [Internet]. Brasília (DF); 2004 [citado 13 de dezembro de 2016]. Disponível em: http://www.planalto.gov.br/ccivil 03/ ato20042006/2004/lei/l10.973.htm

4. Brasil. Lei no 13.243, de 11 de janeiro de 2016. Lei 13.243 de 11 de janeiro de 2016. Dispõe sobre estímulos ao desenvolvimento científico, à pesquisa, à capacitação científica e tecnológica e à inovação e altera a Lei no 10.973 , de 2 de dezembro de 2004, a Lei no 6.815, de 19 de agosto de 1980 , a Lei no 8.666, de 21 de junho de 1993, a Lei no 12.462, de 4 de agosto de 2011, a Lei no 8.745, de 9 de dezembro de 1993, a Lei no 8.958, de 20 de dezembro de 1994, a Lei no 8.010, de 29 de março de 1990, a Lei no 8.032, de 12 de abril de 1990, e a Lei no 12.772, de 28 de dezembro de 2012, nos termos da Emenda Constitucional no 85, de 26 de fevereiro de 2015. [Internet]. Brasília (DF); 2016 [citado 13 de dezembro de 2016]. Disponível em: http://www.planalto.gov.br/ccivil 03/ ato20152018/2016/lei/l13243.htm

5. Brasil. Ministério de Ciência, Tecnologia e Inovação para o Desenvolvimento Nacional. Proposta da Estratégia Nacional de Ciência, Tecnologia e Inovação 2016-2019. Documento para discussão [Internet]. 2015. Disponível em: https://goo.gl/DLwvcF

6. Costa LS, Bahia L, Gadelha CAG. Pensar desenvolvimento e saúde no Brasil. In: Costa, LS; Bahia, L; Gadelha, CAG, organizadores. Saúde desenvolvimento e inovação. Rio de Janeiro: CEPESC; 2015. Vol 2.

7. Viana ALD, Elias PEM. Saúde e desenvolvimento. Ciência \& Saúde Coletiva [Internet]. 2007[citado 2016 nov. 11];12(supl.):1765-77. Disponível em: http://www.scielo.br/pdf/csc/v12s0/02.pdf

8. Viana ALD, Nunes AA, Silva HP. Complexo produtivo da saúde, desenvolvimento e incorporação de tecnologias. In: Ibañez N, Elias PEM, Seixas PHD'A, organizadores. Política e gestão pública em saúde. São Paulo: Hucitec: 2011.vol 1.

9. Emerick MC. Gestão tecnológica como instrumento para a promoção do desenvolvimento econômicosocial: uma proposta para a Fiocruz [dissertação]. Rio de Janeiro: Escola Nacional de Saúde Pública Sérgio Arouca; 2004.

10. Braga PSC. Mapeamento de processos de trabalho da área de contratos e transferência de tecnologia da Gestec como ferramenta para a implementação de melhorias organizacionais [dissertação]. Rio de Janeiro: Escola de Saúde Pública Sérgio Arouca; 2010.

11. Montenegro KBM. Transferência de tecnologia: análise e proposição de estratégia para aperfeiçoar a interação IPPs/universidades-empresas com vistas à inovação em saúde [dissertação]. Rio de Janeiro: Escola de Saúde Pública Sérgio Arouca; 2011.

12. Brasil. Ministério da Saúde. Portaria no 2.376, de 15 de dezembro de 2003 [Internet]. Brasília; 2003. [citado 2016 nov. 16]. Disponível em: http://portal.fiocruz.br/sites/portal.fiocruz.br/files/documentos/ Regimento\%20Interno\%20Fiocruz\%20-\%202003\%20-\%20MS.pdf

13. Fundação Oswaldo Cruz. Portaria 125/86-PR. Cria o Núcleo de Estudos Especiais da Presidência [Internet]. Rio de Janeiro:1986 [citado $2016 \mathrm{dez} 14$ ]. Disponível em: http://www.fiocruz.br/vppis/ gestec/portarias gestec/portaria_125-86 NEP.pdf

14. . Portaria 294/96-PR. Estabelece procedimentos com relação a direitos de propriedade industrial e demais direitos de propriedade sobre invenções ou aperfeiçoamentos passíveis de comercialização, resultantes das atividades realizadas na FIOCRUZ [Internet]. Rio de Janeiro: 1996 [citado 2016 dez 13]. Disponível em: http://www.fiocruz.br/vppis/gestec/portarias gestec/Portaria \%20294-96-Gestec.pdf

15. Portaria 114/98-PR. Subordina a Coordenação de Gestão Tecnológica diretamente à Presidência, desvinculando-a da Assessoria de Planejamento Estratégico. [Internet]. Rio de Janeiro: 1998 [citado 14 de dezembro de 2016]. Disponível em: http://www.fiocruz.br/vppis/gestec/portarias gestec/portaria 114-98-Gestec\%20na\%20Vice.pdf

16. _. Portaria 168/2007-PR. Constitui o Comitê Gestor do Sistema FIOCRUZ de Gestão Tecnológica e Inovação - Sistema GESTEC-NIT, instância colegiada representativa das Unidades Técnico-Científicas e Unidades Técnicas da Fiocruz, vinculado à Coordenação de Gestão Tecnológica. [Internet]. Rio de Janeiro: 2007 [citado 2016 dez 14]. Disponível em: http://www.fiocruz.br/vppis/gestec/portarias gestec/portaria 168 2007.pdf

17. Portaria 133/09-PR. Promover a Gestão Integrada da Inovação na Fiocruz. [Internet]. Rio de Janeiro: 2009 [citado 2016 dez 14]. Disponível em: http://www.fiocruz.br/vppis/gestec/portarias gestec/portaria 133-09 sistema gestec.pdf 
18. Relatório de atividades Gestec 1998. Rio de Janeiro: Multimeios/CICT/Fiocruz; Coordenação de Gestão Tecnológica; 1999.

19. $\ldots$. Relatório de atividades Gestec 2001-2004. Rio de Janeiro: Coordenação de Gestão Tecnológica; 2005.

20. Relatório técnico final do projeto MCT/FINEP 01.06.0777.00. Fortalecimento da gestão tecnológica e da inovação na Fiocruz: implantação do Sistema Gestec-NIT. Período de execução do projeto: 2006-2010 [Internet]. Rio de Janeiro: Coordenação de Gestão Tecnológica; 2011. [citado 2016 nov. 16]. Disponível em: http://www.fiocruz.br/vppis/gestec/sistema gestecnit/Relatorio\%20Final\%20 MCT\%20FINEP\%202006-2010.pdf

21. _. Relatório de Gestão 2008 [Internet]. Rio de Janeiro; 2009. [citado 2016 nov. 16]. Disponível em: http://www.fiocruz.br/media/rel ges 2008.pdf

22. __ _. Relatório de Gestão 2009 [Internet]. Rio de Janeiro; 2010. [citado 2016 nov. 16]. Disponível em: http://www.fiocruz.br/media/relatorio gestao 2009.pdf.

23. _. Relatório de Gestão do exercício de 2010 [Internet]. Rio de Janeiro; 2011. [citado 2016 nov. 16]. Disponível em: http://www.fiocruz.br/media/rel gestao 2010.pdf.

24. Prestação de contas ordinária anual. Relatório de gestão do exercício de 2011[Internet]. Rio de Janeiro: Fiocruz, 2012. [citado 2016 nov. 16]. Disponível em: https://portal.fiocruz.br/sites/portal. fiocruz.br/files/documentos/Relatorio_gestao_2011.pdf

$25 . \quad$. Relatório de gestão do exercício de 2012 [Internet]. Rio de Janeiro; 2013. [citado 2016 nov. 16]. Disponível em: http://portal.fiocruz.br/sites/portal.fiocruz.br/files/documentos/RG_fIOCRUZ_2012_ v01\%20(2).pdf

26. . Relatório de gestão do exercício de 2013 [Internet]. Rio de Janeiro; 2014. [citado 2016 nov. 16]. Disponível em: https://portal.fiocruz.br/sites/portal.fiocruz.br/files/documentos/relatorio_de_ gestao_de_2013_fiocruz_v01.pdf

$27 . \quad$. Relatório de gestão do exercício de 2014 [Internet]. Rio de Janeiro; Fiocruz, 2015. [citado 2016 nov. 16]. Disponível em: https://portal.fiocruz.br/sites/portal.fiocruz.br/files//documentos/relatorio de gestao fiocruz 2014 v15.pdf.

28. Relatório de atividades 2009-2011 [Internet]. Rio de Janeiro; 2012. [citado 2016 nov. 16]. Disponível em: http://www.fiocruz.br/media/relatorio_presidenciarev.pdf

29. . Portfólio de Inovação. Inovação para saúde em sociedade [Internet]. Rio de Janeiro; 2015. [citado 2016 nov. 16]. Disponível em: http://portfolioinovacao.fiocruz.br/?lang=pt

30. Brasil. Lei no 5.772, de 21 de dezembro de 1971. Institui o Código da Propriedade Industrial, e dá outras providências. Diário Oficial da União, Brasília (DF). 1971.

31. Chaves GC, Oliveira MA, Hasenclever L, Melo LM. A evolução do sistema internacional de propriedade intelectual: proteção patentária para o setor farmacêutico e acesso a medicamentos. Cad. Saúde Pública [Internet]. 2007 fev [citado 2016 nov. 16];23(2):257-67. Disponível em: http://www.scielo.br/pdf/csp/ v23n2/02.pdf

32. 32. Gontijo C. As transformações do sistema de patentes, da Convenção de Paris ao acordo TRIPS: a posição brasileira [Internet]. Berlin: Fundação Heinrich Böll; maio 2005 [citado 2016 nov. 16]. Disponível em: http://www.fdcl-berlin.de/fileadmin/fdcl/Publikationen/C cero-FDCL.pdf

33. Brasil. Lei no 9.279 de 14 de maio de 1996. Regula direitos e obrigações relativos à propriedade industrial. [Internet]. Brasília (DF); 1996 [citado 13 de dezembro de 2016]. Disponível em: http://www. planalto.gov.br/ccivil 03/leis/L9279.htm

34. Perfil dos integrantes do Sistema Gestec NIT [Internet]. Rio de Janeiro: Coordenação de Gestão Tecnológica; 2014. [citado 2016 nov. 16]. Disponível em: http://www.fiocruz.br/vppis/gestec/docs/ experiencia.equipe.envolvida.2014.pdf.

35. Sistema Fiocruz de Gestão Tecnológica e Inovação. Cursos [Internet]. Rio de Janeiro; 2015. [citado 2016 nov. 16]. Disponível em: http://www.fiocruz.br/vppis/gestec/cursos.php

36. Sistema Fiocruz de Gestão Tecnológica e Inovação. Cursos anteriores [Internet]. Rio de Janeiro; 2013. [citado 2016 nov. 16]. Disponível em: http://www.fiocruz.br/vppis/gestec/cursos2013.php 
37. S. Sistema Fiocruz de Gestão Tecnológica e Inovação. Eventos organizados pelo Sistema GestecNIT/ Eventos apoiados pelo Sistema Gestec-NIT/[Internet]. Rio de Janeiro; 2015. [citado 2016 nov. 16]. Disponível em: http://www.fiocruz.br/vppis/gestec/eventos.php.

38. _ Sistema Fiocruz de Gestão Tecnológica e Inovação. CNPq aprova projeto que contempla Sistema Gestec-NIT[Internet]. Rio de Janeiro; 2014. [citado 2016 nov. 16]. Disponível em: http://www. fiocruz.br/vppis/gestec/noticias.php

39. Montenegro, KBM, Carvalho, CLC. Guia de ações preventivas em propriedade intelectual, informação tecnológica e transferência de tecnologia para geradores de conhecimento. Sistema Fiocruz de Gestão Tecnológica e Inovação. [Internet]. Fiocruz, Rio de Janeiro; 2015.[citado 2016. nov. 16]. http://www. fiocruz.br/vppis/gestec/docs/guia acoes prev.pdf 